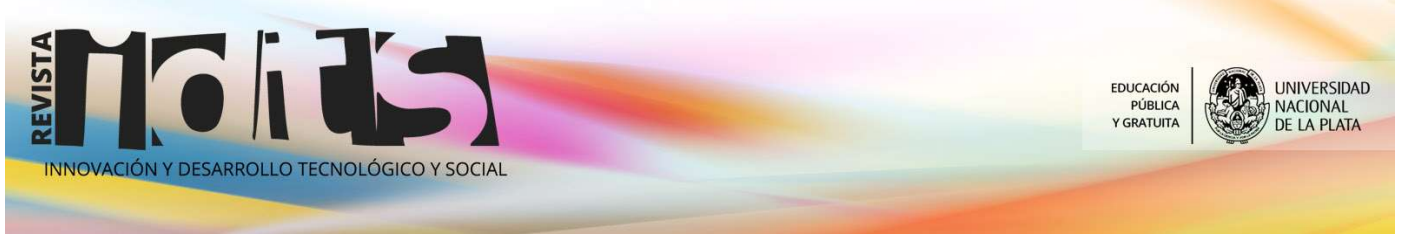

Innovación y Desarrollo Tecnológico y Social (2019) 1 (1): 52-60

\title{
Aportes a la conservación poscosecha de flores de corte para productores del Gran La Plata
}

\author{
Hasperué Joaquin ${ }^{1}$, Nico Andres², Riccione Julieta², Cieza Ramón ${ }^{23}$ \\ 1. Laboratorio de Investigación en Productos Agroindustriales (LIPA), Facultad de Cs. Agrarias y \\ Forestales, Universidad Nacional de La Plata (UNLP) \\ 2.Facultad de Ciencias Agrarias y Forestales (FCAyF), UNLP. ${ }^{3}$ cieza@agro.unlp.edu,ar
}

Resumen. El Partido de La Plata y localidades vecinas constituyen la región de mayor producción de flores de corte de Argentina, la cual abastece de una amplia diversidad de especies florales a distintos puntos del país. La producción la realizan principalmente productores de tipo familiar, generando ingresos y mano de obra para la región. Uno de los problemas de mayor relevancia para los productores florícolas es la pérdida de calidad comercial de las varas florales después de la cosecha. La utilización de cámaras frigoríficas permite mantener la calidad comercial de las flores mejorando así su capacidad de negociación manteniendo el producto en contextos de bajo precio. Sin embargo, sólo un bajo porcentaje de productores cuentan con esta tecnología, dado que es costosa para su adquisición y funcionamiento operativo. Poder avanzar en tecnologías de conservación que reduzcan las pérdidas poscosecha y generen un mayor ingreso a los productores es uno de los desafíos para un sector que adolece en este tipo de tecnologías. Este trabajo describe un proyecto generado desde la Facultad de Ciencias Agrarias y Forestales con financiamiento del Programa "Agregando Valor" de la Secretaría de Políticas Universitarias de la Nación, en el cual se ensayaron tecnologías sencillas de bajo costo para la conservación poscosecha de flores de corte. El mismo surge de la demanda de los productores a través de un grupo de Cambio Rural (INTA-MINAGRO) y del mercado cooperativo de Flores (MERCOFLOR). Se buscó mejorar la conservación poscosecha de Alstroemeria x hybrida y Lilium longiflorum, especies cuyo cultivo se ha incrementado en los últimos años en la región y que presentan como problema poscosecha más visible el amarilleamiento de las hojas de la vara. Para ello, se utilizaron distintas fuentes de iluminación LED: blanco cálido o rojo+azul, siendo los controles almacenados en oscuridad. La intensidad de las fuentes se fijó en $50 \mu \mathrm{mol} \mathrm{m} \mathrm{m}^{-2} \mathrm{~s}^{1}$, con un periodo de luz de $16 \mathrm{~h}$. La temperatura de almacenamiento fue de $21^{\circ} \mathrm{C}$ y la humedad relativa (HR) de $70 \%$. El tratamiento con ambos tipos de luz LED logró un aumento de la vida útil de las varas de Alstroemeria sp. en 7 días. Por otra parte, para evaluar el efecto hormonal en el retraso del amarilleamiento, se realizó en otro experimento un pulsado por $24 \mathrm{~h}$ en 50ppm de ácido gibérelico $\left(A G_{3}\right)$ en varas de Alstroemeria $s p$. y Lilium $s p$. pasando luego a agua destilada $\mathrm{pH}=3,5$ a $21^{\circ} \mathrm{C}$ y $70 \%$ HR. Se observó una mejor conservación de las varas en los tratamientos con $A G_{3}$ con respecto al testigo en ambas especies a los días 7 y 14. Se concluye que las técnicas desarrolladas en este proyecto, de fácil apropiación por los productores familiares de la región, podrían generar un impacto positivo en el retraso del amarilleamiento y mejora de la calidad poscosecha de las flores de corte. Los resultados obtenidos fueron presentados en una jornada demostrativa en MERCOFLOR ante productores y floristas. Se realizaron cartillas informativas con las tecnologías ensayadas y recomendaciones prácticas para su implementación.

Palabras clave: floricultura; calidad comercial; poscosecha; ácido giberélico; luz LED

Recibido: 29/04/2019 Aceptado: 03/07/2019

DOI: https://doi.org/10.24215/26838559e004 


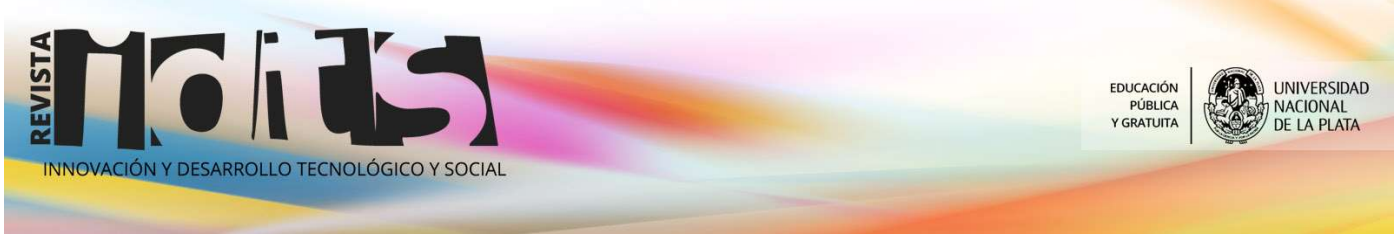

Innovación y Desarrollo Tecnológico y Social (2019) 1 (1): 52-60

\title{
Contributions to the post-harvest conservation of cut flowers for producers of La Plata city
}

\begin{abstract}
La Plata city and neighboring towns constitute the region with the highest production of cut flowers in Argentina, which supplies a wide variety of floral species to different parts of the country. Production is mainly carried out by family-type producers, generating income and labor for the region. One of the most relevant problems for flower growers is the loss of commercial quality of the flower rods after harvest. The use of cold rooms allows to maintain the commercial quality of the flowers and allows a sales delay, thus improving their bargaining ability while maintaining the product in low price contexts. However, only a small percentage of producers have this technology, given that it is expensive for its acquisition and operational functioning. The improvement of conservation technologies conducive to a decrease postharvest losses and thus to a greater income is one of the most important challenges for the sector that lacks this type o technology. This work describes a project generated from the Facultad de Cs. Agrarias y Forestales funded by the "Adding Value" Program of the Secretaría de Politicas Universitarias de la Nación, in which simple low-cost technologies for the postharvest conservation of cut flowers were tested. This project arises from the demand of the producers through a group of "Cambio Rural" (INTA-MINAGRO) and the "Mercado Cooperativo de Flores" (MERCOFLOR). We sought to improve the postharvest conservation of Alstroemeria $x$ hybrida and Lilium longiflorum flowers, species whose cultivation has been increased in the region in recent years and which present the yellowing of the leaves of the rod as the most visible post-harvest problem. For this reason, different sources of LED lighting were used: warm white or red + blue, with controls being stored in darkness. The intensity of the sources was set at $50 \mu \mathrm{mol} \mathrm{m} \mathrm{m}^{-2} \mathrm{~s}^{-1}$, with a light period of $16 \mathrm{~h}$. The storage temperature was $21^{\circ} \mathrm{C}$ and the relative humidity $(R H)$ was $70 \%$. The treatment with both types of $\angle E D$ light achieved an increase in the shelf life of the rods up to 7 days. On the other hand, to evaluate the hormonal effect in the leave's yellowing delay, a $24 \mathrm{~h}$ pulse was made with a $50 \mathrm{ppm}$ gibberelic acid (AG) in Alstroemeria sp. and Lilium sp. rods, which were subsequently stored in distilled water $p H=3.5$ at $21{ }^{\circ} \mathrm{C}$ and $70 \% \mathrm{RH} . \mathrm{A}$ better conservation of the rods was observed in the $\mathrm{AG}_{3}$ treatments compared to controls in both species at 7 and 14 days of storage. It is concluded that the techniques developed in this project, which could be easily appropriated by the family producers of the region, could generate a positive impact on the delay of yellowing and an improvement of the post-harvest quality of the cut flowers. The results obtained were presented in a demonstration day in MERCOFLOR for producers and florists. Information sheets were made with the tested technologies, containing practical recommendations for their implementation.
\end{abstract}

Keywords: floriculture; commercial quality; postharvest; gibberellic acid; LED light 


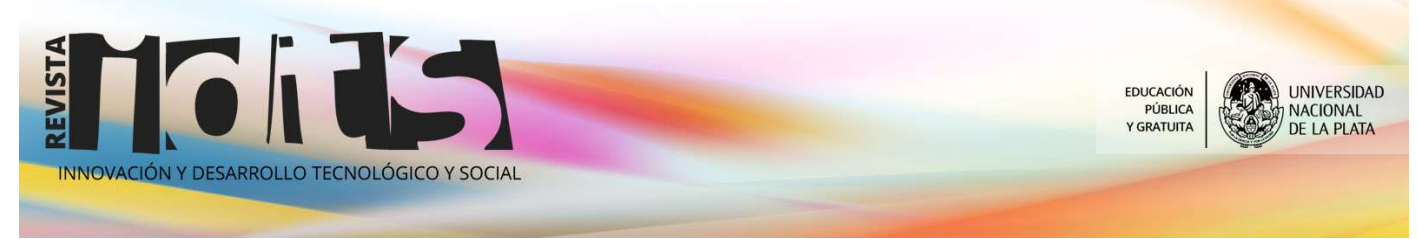

Innovación y Desarrollo Tecnológico y Social (2019) 1 (1): 52-60

\section{Novedad u originalidad local en el conocimiento:}

La producción argentina de flores de corte se concentra en el sur del Área Metropolitana de Buenos Aires, destacándose el Partido de La Plata respecto a superficie productiva y volumen de producción. De acuerdo a los datos censales, el mismo concentraba el $64 \%$ de la producción de La Provincia de Buenos Aires, en su gran mayoría proveniente de productores de carácter familiar (Cieza, 2014). Este Partido constituye, por otra parte, el más importante polo productivo de cultivos intensivos bajo cubierta del país, que se concentra en la producción de hortalizas y flores de corte.

En el presente trabajo se utilizaron tecnologías de fundamento físico y bioquímico para la conservación de flores de corte, tomando como base antecedentes de tecnologías formuladas para retrasar la senescencia y que actualmente no están difundidas ni adaptadas por los productores del partido de La Plata. Estas tecnologías se probaron y adaptaron a especies florícolas determinadas tomando en cuenta el escenario actual de los productores regionales. Se realizó una primera instancia de interacción con los productores mediante una reunión grupal en MERCOFLOR, a fin de identificar, a partir de sus demandas, cuáles eran las especies para las cuales presentaba mayor interés la utilización de determinadas tecnologías para preservar la calidad y en qué etapas de la cadena de comercialización resultaba más apropiada su aplicación. La investigación en tecnología de conservación poscosecha de flores de corte se encuentra en sus etapas iniciales de desarrollo y cualquier aporte al mismo puede redundar en beneficios para el sector.

\section{Grado de relevancia:}

Una de las principales problemáticas a las que deben enfrentarse los productores florícolas es la que se asocia a la comercialización. A menudo la amplia tecnología disponible, una conducción correcta y la oportuna aplicación de las prácticas culturales permiten obtener a la cosecha varas de aceptable calidad, de acuerdo con los patrones exigidos por el mercado. Sin embargo, a partir del momento de la cosecha, diversas causas asociadas a la conservación del producto, determinan que en poco tiempo disminuya su calidad cosmética, y con ella su valor de venta, lo cual desemboca en muchos casos en el descarte de la mercadería. Por otra parte, algunas variables ambientales llevan a que la floración se concentre en un periodo corto de tiempo, aumentando la oferta y saturando el mercado en determinados periodos con una sensible baja en el precio de venta. A esto contribuyen, por otra parte, las condiciones particulares de la demanda de este tipo de productos, que exigen al floricultor concentrar la oferta para abastecer al consumidor en fechas clave de venta como las celebraciones por el día de la madre, etc., donde la demanda es mayor. Si bien se utilizan en algunos casos las cámaras frigoríficas, las mismas son costosas y el gasto operativo en electricidad es elevado. Por otra parte el desarrollo y la adopción de otro tipo de tecnologías que permitan una mayor vida útil de los productos florícolas son incipientes e insuficientes. 


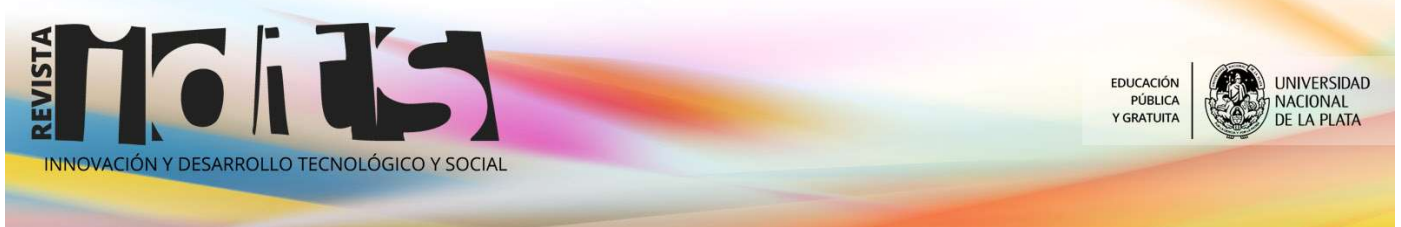

Innovación y Desarrollo Tecnológico y Social (2019) 1 (1): 52-60

\section{Grado de pertinencia:}

El desarrollo generado permite con técnicas simples y relativamente económicas plantear estrategias de conservación de flores utilizando herramientas y productos que se encuentran accesibles para los productores. Por otra parte podría suplantar a soluciones preservantes comerciales que cuentan como desventaja su alto costo y, en ocasiones, su toxicidad que anula su aceptación en potenciales destinos de exportación (De la Riva Morales, 2011). La tecnología ensayada incorpora la aplicación de hormonas vegetales (ácido giberélico, $\mathrm{AG}_{3}$ ) aplicadas en forma de pulsado y la utilización de luces LED. Ambas tecnologías son de fácil acceso pudiéndose adquirir en locales comerciales de la región. Por otra parte, las luces cuentan con una sencilla aplicación y su costo de adquisición y consumo es bajo, al igual que la utilización de $\mathrm{AG}_{3}$, en comparación a otros preservantes comerciales. En nuestros ensayos, las tecnologías fueron probadas en dos especies florícolas (Lilium longiflorum var. Litouwen y Alstroemeria x hybrida), y se obtuvo una prolongación en la vida útil, perceptible tanto por un retraso en la caída de los pétalos como por una reducción del amarilleamiento de las hojas. Investigaciones en poscosecha de hortalizas (Hasperué y col., 2016 a,b) mostraron aspectos positivos de la aplicación de luces LED en la mejora de la calidad comercial. Sin embargo, actualmente no hay desarrollos de estas tecnologías para el cultivo comercial de flores.

\section{Grado de demanda:}

La problemática de la poscosecha en flores fue planteada por el Diagnóstico Florícola (INTA 2005), en la que se relevó por medio de encuestas la demanda de los actores de la cadena. En la misma se expresaba que no había un conocimiento de soluciones preservantes y un tratamiento adecuado según las especies. El Clúster Florícola del AMBA y San Pedro constituido por los Mercados Florícolas, INTA, y organizaciones de productores plantearon como uno de los temas prioritarios de mejora la poscosecha de flores de corte (PROSAP, 2012). En el año 2016 desde el Grupo de Cambio Rural II "Asociación de Productores Las Banderitas" se manifestó la necesidad de desarrollar tecnologías que mejoren la conservación de las flores, dada la gran cantidad de varas descartadas debido a la pérdida de calidad comercial, con la consiguiente repercusión negativa en la renta de los productores. Este diagnóstico fue coincidente con el del Mercado Cooperativo de Flores (MERCOFLOR), que también la consideró un tema de relevancia y preocupación entre los socios. En este sentido en el año 2016 se realizó la presentación del proyecto "Conservando flores de calidad" del programa "Agregando Valor" de la Secretaria de Políticas Universitarias. El mismo se inició en marzo de 2017 y concluyó en diciembre de 2018.

\section{Desarrollo del producto}

En los experimentos que se presentan en este informe se buscó mejorar la conservación poscosecha de Alstroemeria sp., y Lilium sp., dos importantes especies cultivadas en la región, que presentan como inconveniente principal el amarilleamiento 
de las hojas de la vara durante el almacenamiento. Para ello, en uno de los experimentos, se utilizaron dos fuentes de iluminación LED (blanco cálido y rojo+azul) mientras que los controles fueron almacenados en oscuridad. En base a estudios previos la intensidad de iluminación se fijó en $50 \mu \mathrm{mol} \mathrm{m} \mathrm{m}^{-2} \mathrm{~s}^{-1}\left(\approx 10,45 \mathrm{~W} / \mathrm{m}^{2}\right)$, con un tiempo de iluminación de $16 \mathrm{~h}$. La temperatura de almacenamiento fue de $21^{\circ} \mathrm{C}$ y la humedad relativa de $70 \%$.

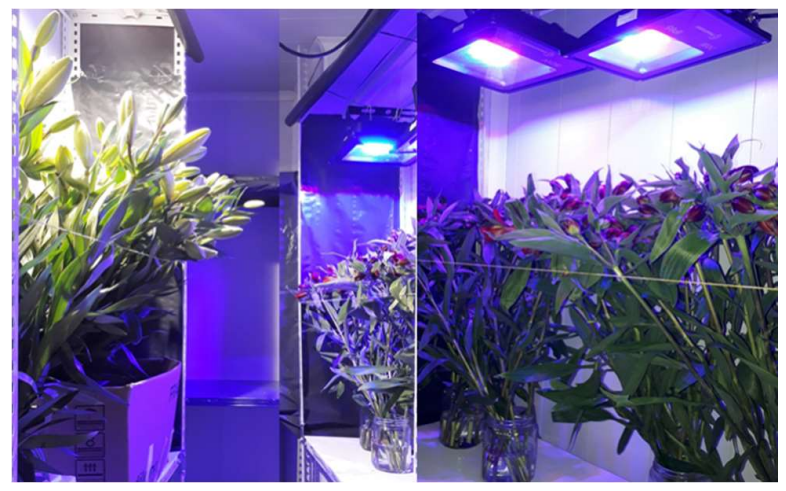

Figura 1. Tratamientos con iluminación. Las irradiancias se ajustaron con la distancia de las fuentes de luz a las varas florales. / Figure 1. Light treatments. The irradiances were set by adjusting the distance between the flowers and the light sources.

Las hojas de las varas almacenadas bajo el régimen de iluminación se observaron notablemente más verdes tanto a los siete como a los catorce días de almacenamiento. Éste fenómeno se confirmó de manera objetiva a través de la determinación colorimétrica y la medición de la concentración de clorofila en hojas. La caída de los tépalos de las flores también se retrasó con el tratamiento. No hubo diferencias entre las muestras almacenadas en luz LED blanca cálida respecto de las almacenadas en luz rojo + azul. El tratamiento logró aumentar la vida útil de las varas 7 días, dado que las varas tratadas llegaron al D14 de almacenamiento en buen estado de conservación mientras que los controles mostraron amarilleamiento al D7. 


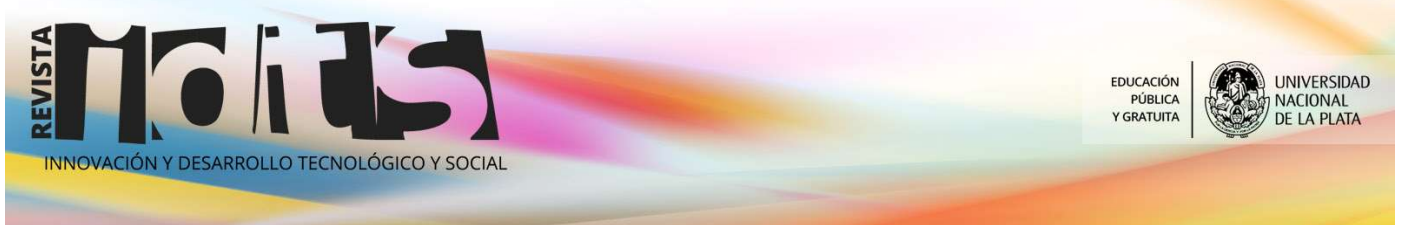

Innovación y Desarrollo Tecnológico y Social (2019) 1 (1): 52-60
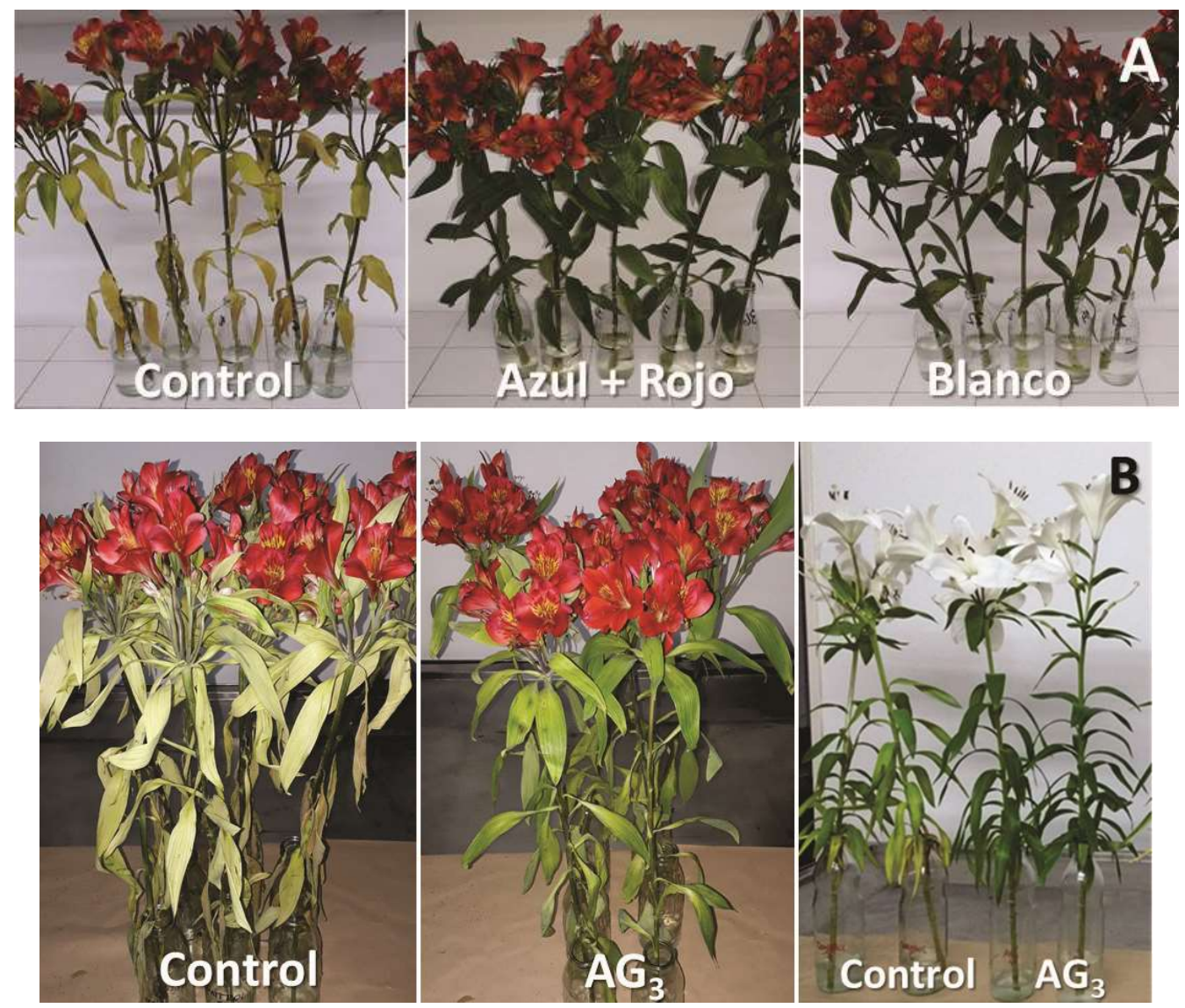

Figura 2. Flores expuestas a iluminación $L E D$ durante el almacenamiento $(A)$ y sometidas a un pulso de 24 h de ácido giberélico $\left(A G_{3}\right)$, almacenadas a $21^{\circ} \mathrm{C}(B)$. Las imágenes $A$ y $B$ se corresponden con el día 14 de almacenamiento. / Figure 2. Cut flowers exposed to LED illumination throughout the storage (A) and treated with a pulse of giberellic acid $\left(A G_{3}\right)$ and stored at $21^{\circ} \mathrm{C}(B)$. Images $A$ and $B$ are referred as day 14 of storage.

En el otro experimento, destinado a evaluar el efecto de giberelinas sobre la poscosecha, se efectuó un pulsado (inmersión temporal del extremo proximal de las varas previa a la conservación) con una solución de $50 \mathrm{ppm}$ de ácido giberélico $\left(\mathrm{AG}_{3}\right)$ durante 24 horas y bajo condiciones de iluminación similares a las del experimento con luz LED únicamente durante el periodo del pulsado. Tanto las varas pulsadas como los controles se conservaron posteriormente en agua destilada con ajuste de $\mathrm{pH}$ a 3,5 con ácido cítrico (solución de conservación), a $21^{\circ} \mathrm{C}$ y $70 \%$ de humedad relativa. El deterioro de las varas durante el almacenamiento se hizo evidente por un mayor amarilleamiento de las hojas tanto en Alstroemeria sp. como en Lilium sp. Sin embargo, al cabo de 14 días de conservación, la reducción del color verde y aumento del color amarillo (medida a través de los parámetros de color Hue y $L^{*}$ respectivamente) en Alstroemeria sp. fue menor en las varas pulsadas con $\mathrm{AG}_{3}(9,3 \%)$ respecto de los controles sin pulsar $(61,6 \%)$. El mismo efecto se observó en las varas de Lilium sp., aunque de manera menos evidente. 


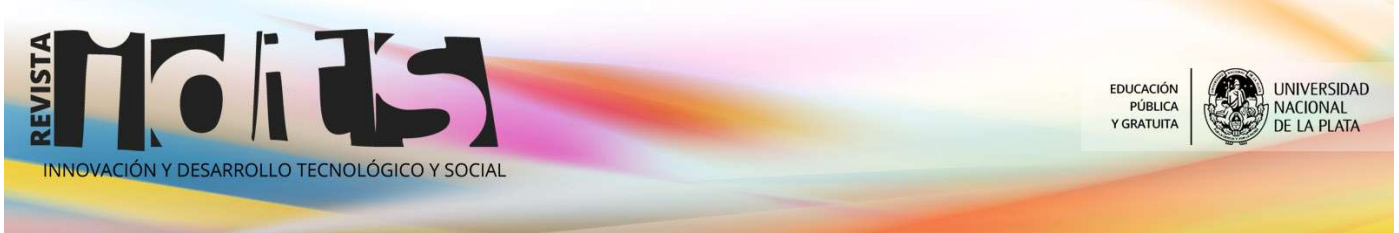

Innovación y Desarrollo Tecnológico y Social (2019) 1 (1): 52-60

Tabla 1. Desarrollo de color. $L^{*}$ : luminosidad ( $L^{*}=0$, negro; $L^{*}=100$, blanco), intensidad del desarrollo del amarilleamiento foliar; Hue: tono, índice de verdor de las hojas (el valor, ángulo del tono, se expresa en grados que van de $0^{\circ}$ a $360^{\circ}$, valores bajos indican menor tonalidad verde) ; $C=$ control, $B C O=$ blanco cálido, $A+R=$ luz azul y rojo); $S D=$ desvío estándar. / Table 1. Color development. $L^{*}$ : luminosity ( $L^{*}=0$, black; $L^{*}=100$, white), intensity of leave yellowing; Hue: leaf greenness index (hue angle is expressed in degrees ranging from $0^{\circ}$ to $360^{\circ}$, low values indicate lower greenness); $C=$ control, $B C O=$ warm white, $A+R=$ blue and red light; $S D=$ standard deviation.

\begin{tabular}{cccccc}
\hline & \multicolumn{2}{c}{$\mathrm{L}^{*}$} & \multicolumn{2}{c}{ 'HUE (tono) } \\
& & Media & SD & Media & SD \\
\hline D0 & C & $36,09 \mathrm{a}$ & 2,18 & $127,93 \mathrm{de}$ & 1,63 \\
& BCO & $36,09 \mathrm{a}$ & 2,18 & $127,93 \mathrm{de}$ & 1,63 \\
& A+R & $36,09 \mathrm{a}$ & 2,18 & $127,93 \mathrm{de}$ & 1,63 \\
& & & & & \\
D7 & C & $51,95 \mathrm{~d}$ & 4,58 & $116,68 \mathrm{~b}$ & 3,10 \\
& BCO & $40,23 \mathrm{~b}$ & 1,99 & $126,25 \mathrm{de}$ & 1,60 \\
& A+R & $39,99 \mathrm{~b}$ & 2,00 & $126,96 \mathrm{~d}$ & 1,95 \\
& & & & & \\
D14 & C & $65,05 \mathrm{e}$ & 2,70 & $104,64 \mathrm{a}$ & 3,31 \\
& BCO & $42,88 \mathrm{c}$ & 6,66 & $124,45 \mathrm{~d}$ & 5,48 \\
& A+R & $42,05 \mathrm{c}$ & 2,54 & $126,14 \mathrm{c}$ & 2,29 \\
\hline
\end{tabular}

Tanto el almacenamiento con luz LED como con $\mathrm{AG}_{3}$ (con una adaptación adecuada a cada establecimiento productor o comercializador de flores), así como un tratamiento que contemple la aplicación de ambas variables en conjunto, resultarían alternativas económicas para retrasar el proceso de senescencia y amarilleamiento foliar en Alstroemeria sp. y en Lilium sp., alargando en días la viabilidad del producto. De no contarse con cámara de frío, es factible disponer las fuentes de iluminación LED en una estructura de soporte que ilumine preferentemente las varas desde arriba, utilizando luxómetro o sensor PAR para ajustar la dosis adecuada de luz. En cuanto a la aplicación de $\mathrm{AG}_{3}$, es factible la preparación de la solución a la concentración recomendada y de $\mathrm{pH}$ ajustado, en baldes donde se les aplicará un pulso de $24 \mathrm{~h}$ en presencia de luz para favorecer la transpiración de la vara y el arrastre de la solución del balde a través de ella. 
Innovación y Desarrollo Tecnológico y Social (2019) 1 (1): 52-60

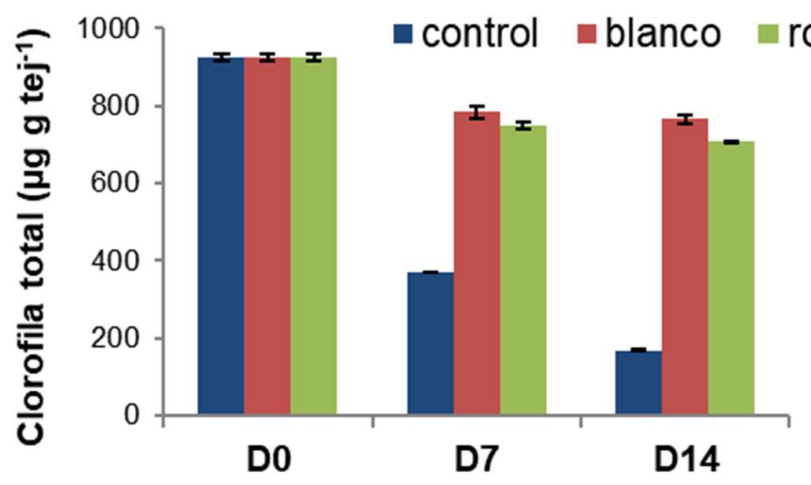

Figura 3. Contenido de clorofila en hojas de varas florales almacenadas en oscuridad (control) $y$ sometidas a tratamientos con luz blanca y rojo+azul $\left(50 \mu \mathrm{mol} \mathrm{m} \mathrm{m}^{-2} \mathrm{~s}^{-1}\right)$. / Figure 3. Chlorophyll content in leaves of flower rods stored in the dark (control) and treated with white and red+blue light (50 $\mu \mathrm{mol} \mathrm{m}^{-2}$ $\left.s^{1}\right)$.

Tono

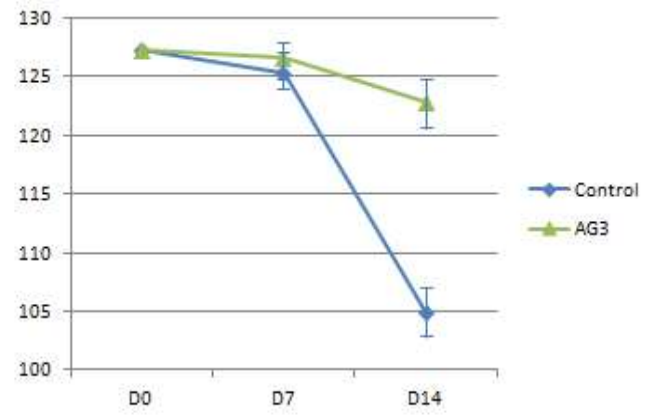

Luminosidad

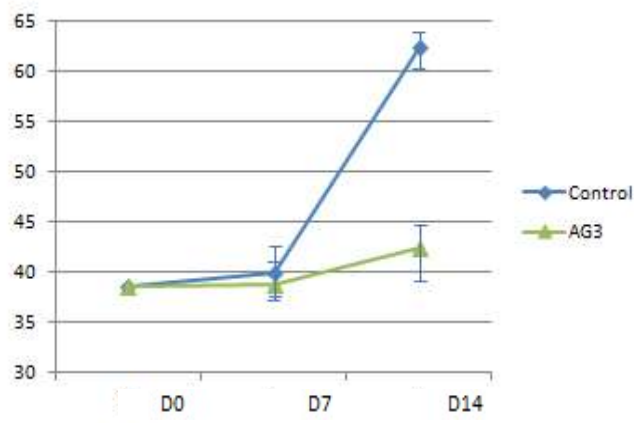

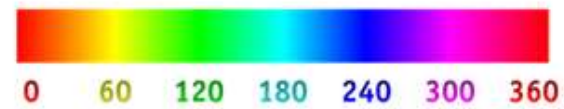

Figura 4. Evolución de los parámetros de color en hojas de varas de Alstroemeria sp. mantenidas a 21 ${ }^{\circ} \mathrm{C}$, y fotoperíodo de 16 h luego de someterlas a pulsado con agua destilada (Control) y ácido giberélico $\left(A G_{3}\right)$. Se adjunta la barra de color con los valores correspondientes de hue a lo largo de la escala para facilitar la interpretación de los valores del gráfico. La barra alrededor de los puntos que señalan el valor en las curvas indican el intervalo de confianza para una separación de medias por el método de la mínima diferencia significativa $(P \geq 0.05)$. / Figure 4. Evolution of colour parameters in leaves from Alstroemeria sp. rods, stored at $21^{\circ} \mathrm{C}$ and $16 \mathrm{~h}$ day-length, after having been pulsed with distilled water (Control) and gibberelic acid $\left(A G_{3}\right)$. Colour bar with hue scale is attached, in order to compare with graphics data. The bars around the points of value in the curves indicate the confidence interval for means comparison by $\angle S D$ method $(P \geq 0.05)$. 


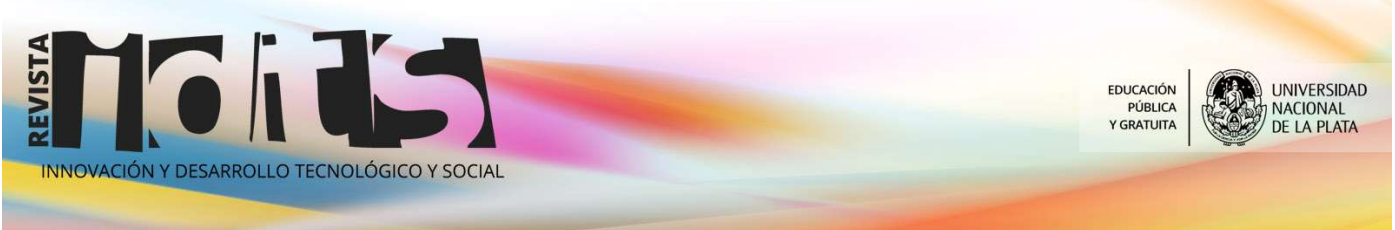

Innovación y Desarrollo Tecnológico y Social (2019) 1 (1): 52-60

\section{Financiamiento}

Proyectos de Vinculación Tecnológica "Universidades Agregando Valor" VT12UNLP4783 - Proyecto "Conservando flores de calidad". Ejecución 2017-2018

\section{Agradecimientos}

Cooperativa Mercoflor. Ing. Agr. Gerardo Rambeaud.

Asociación de Productores Las Banderitas

Agencia de Extensión Rural INTA El Pato

Instituto de Floricultura de Castelar.

\section{Referencias bibliográficas}

Cieza. R. 2014. Caracterización de la producción florícola en el Partido de La Plata. En Revista de la Facultad de Agronomía. Vol 113 (1) pp 28-37 Facultad de Ciencias Agrarias y Forestales, UNLP

Hasperué, J. H., Guardianelli, L., Rodoni, L. M., Chaves, A. R., \& Martínez, G. A. (2016a). Continuous white-blue LED light exposition delays postharvest senescence of broccoli. LWT-Food Science and Technology, 65, 495-502.

Hasperué, J. H., Rodoni, L. M., Guardianelli, L. M., Chaves, A. R., \& Martínez, G. A. (2016b). Use of LED light for Brussels sprouts postharvest conservation. Scientia Horticulturae, 213, 281-286.

INTA 2005. Plan Tecnológico Regional 2006-2008. Informe diagnóstico de situación Cadena Florícola

De la Riva Morales, F. 2011. Poscosecha de flores de corte y medio ambiente. En IDESIA (Chile) Volumen 29, № 3. Páginas 125-130 Septiembre-Diciembre, 2011

PROSAP. 2012. Informe de reunión de equipo técnico. Presentado en asamblea del Cluster Floricola de AMBA y San Pedro. Inédito. 